\title{
Nucleotide sequence analysis and genomic organization of the NY-RPV isolate of barley yellow dwarf virus
}

\author{
Jeffrey R. Vincent,* Richard M. Lister and Brian A. Larkins $\dagger$ \\ Department of Botany and Plant Pathology, Purdue University, West Lafayette, Indiana 47907-1155, U.S.A.
}

cDNA clones representing the ssRNA genome of the NY-RPV isolate of barley yellow dwarf luteovirus (BYDV) were sequenced and 5600 nucleotides of the genome were determined. The deduced genome organization has limited similarity to that of another BYDV isolate, Vic-PAV, but is identical to that of beet western yellows (BWYV) and potato leafroll (PLRV) luteoviruses. NY-RPV has six major positive-sense open reading frames (ORFs) and, by comparison with RNA-dependent RNA polymerase and nucleic acid helicase consensus sequence motifs, it is postulated that NY-RPV ORF2 and ORF3 encode the viral replicase, which is expressed by a translational frameshift mechanism. The region of the NY-RPV genome containing the $22 \mathrm{~K}$ coat protein $\mathrm{ORF}$, the apparently associated internal apparent VPg ORF and the ORF immediately 3'-proximal (ORF6) to the coat protein ORF are organized as reported for other luteoviruses. Evidence is presented showing that ORF6 is expressed by readthrough of the coat protein gene termination codon, and that this protein is associated with the intact virus as a $65 \mathrm{~K}$ protein. Although NY-RPV infects graminaceous rather than dicotyledonous plants, the taxonomic relationships between BYDV isolates and other luteoviruses deduced from the genome organization and sequence data strongly suggest that NY-RPV is distinct from the PAV-like isolates of BYDV and is more closely related to BWYV and PLRV.

\section{Introduction}

Luteoviruses, such as barley yellow dwarf virus (BYDV), beet western yellows virus (BWYV) and potato leafroll virus (PLRV), cause yellowing diseases in a wide range of host plants (Matthews, 1982), are not mechanically transmitted and are limited to the phloem tissue of the host plant. The physical properties of luteoviruses are similar in that they are 24 to $30 \mathrm{~nm}$ diameter isometric particles containing a positive-sense ssRNA genome of 5.6 to $6.0 \mathrm{~kb}$ (Mayo et al., 1989; Miller et al., 1988a; Veidt et al., 1988; Waterhouse et al., 1988). The genomic RNA has a small protein $(\mathrm{VPg})$ covalently attached to its $5^{\prime}$ end (Murphy et al., 1989) but does not have a poly(A) tail (Mayo et al., 1982, 1989; Miller et al., 1988 a; Veidt et al., 1988).

Unlike BWYV and PLRV, which typically infect dicotyledonous hosts, BYDV consists of a group of serologically related viruses that infect barley, oats, wheat, rice, maize and other graminaceous hosts (Rochow, 1970). Like other luteoviruses, they are

† Present address: Department of Plant Science, University of Arizona, Tucson, Arizona 85721, U.S.A.

The nucleotide sequence data reported in this paper will appear in the DDJB, EMBL and GenBank nucleotide sequence databases under the accession number D01013. transmitted only by aphid vectors in a persistent, circulative manner. Isolates of BYDV, originally distinguished and named for their predominant aphid vectors, have been separated into two groups based on their serological relationships (Aapola \& Rochow, 1971; Rochow, 1970; Rochow \& Carmichael, 1979; Rochow \& Duffus, 1981), the cytopathological ultrastructure of infected cells (Gill \& Chung, 1970) and the dsRNA profiles identified in infected tissues (Gildow et al., 1983). Based on these criteria, BYDV group 1 includes serotypes MAV, transmitted by Macrosiphon (=Sitobion) avenae Fabr., PAV, transmitted by $M$. avenae and Rhopalosiphum padi $\mathrm{L}$., and SGV, transmitted by Schizaphis graminum Rond. (Rochow, 1970). Group 2 includes serotypes RMV, transmitted by $R$. maidis Fitch., and RPV, transmitted by $R$. padi (Rochow, 1970).

To help understand the molecular biology of luteoviruses, the nucleotide sequence and genome organization of a PAV serotype of BYDV (Miller et al., 1988a), and BWYV (Veidt et al., 1988) and PLRV (Mayo et al., 1989; Keese et al., 1990) have been determined. The coat protein genes of these luteoviruses (Miller et al., 1988b; Kawchuk et al., 1989; Prill et al., 1989; Veidt et al., 1988), and of isolates representing the two BYDV groups described have also been identified and characterized 
(Vincent et al., 1990). Here, we present the nucleotide sequence and genome organization of the RPV serotype of BYDV. This information is compared to that for a PAV serotype of BYDV, and also to that published for BWYV and PLRV. Analyses of sequence similarity and genome relationships are presented which indicate that the RPV serotype, although it infects and causes yellowing disease in graminaceous hosts, is more closely related to BWYV and PLRV, which typically infect dicotyledonous hosts, than to PAV serotypes of BYDV.

\section{Methods}

Virus and RNA isolation. The BYDV isolate used in this work was the New York RPV isolate of Rochow (NY-RPV) (Rochow, 1970), named according to the suggestion of Rochow (1984) that isolates originating in New York State be prefixed by 'NY'. This isolate was maintained at Purdue University in oat plants (Avena sativa L. cv. Clintland 64) by mass transfer of viruliferous $R$. padi; the absence of cross-contamination was confirmed by ELISA as described previously (Barbara $e t$ al., 1987). Virus was purified and the RNA extracted as described for the MAV-PS1 isolate of BYDV (Barbara et al., 1987).

CDNA. A cDNA library representing the NY-RPV genome was constructed in $\lambda$ gt1l (Young \& Davis, 1983), pUC18 (Vieira \& Messing, 1982) and pGEM-3Z (Promega) (Barbara et al., 1987; Vincent et al., 1990). A restriction map representing the viral genome was generated by single and double restriction enzyme digests of the cloned cDNA, and by Southern blot hybridization between different restriction fragments (Maniatis et al., 1982).

$c-D N A$ sequencing. Plasmid DNA isolated by an alkaline lysis technique (Birnboim \& Doly, 1979) was sequenced by the dideoxynucleotide chain termination method (Sanger et al., 1977) with a modified T7 DNA polymerase (Sequenase; U.S. Biochemical). The cDNA clones representing the viral genome were sequenced by one or more of the following strategies. (i) Restriction fragments representing overlapping regions of the genome were subcloned; (ii) exonuclease III/ mung bean nuclease-generated nested deletions were identified in existing cDNA clones (Putney et al., 1981); (iii) specific synthetic sequencing primers were prepared (Laboratory for Macromolecular Structure, Department of Biochemistry, Purdue University). Sequence analyses were performed using Microgenie version 4.0 (Beckman Instruments) and the Genetics Computer Group sequence analysis software, version 6.1 (Devereux et al., 1984).

Western blot analysis. For Western blot analysis of NY-RPV, purified virus particles were dissociated by the addition of an equal volume of $2 \times$ SDS-PAGE sample buffer (Laemmli, 1970), incubated overnight at $4{ }^{\circ} \mathrm{C}$ and electrophoresed on a $10 \%$ polyacrylamide gel. Viral proteins were detected immunologically with $0.15 \%$ Tween 20 used to prevent non-specific binding (Barbara et al., 1987).

\section{Results and Discussion}

\section{Sequencing}

Overlapping cDNA clones representing NY-RPV genomic RNA were identified by restriction enzyme analysis and hybridization (Fig. 1). From them, 5600 nucleotides of the NY-RPV genome were determined

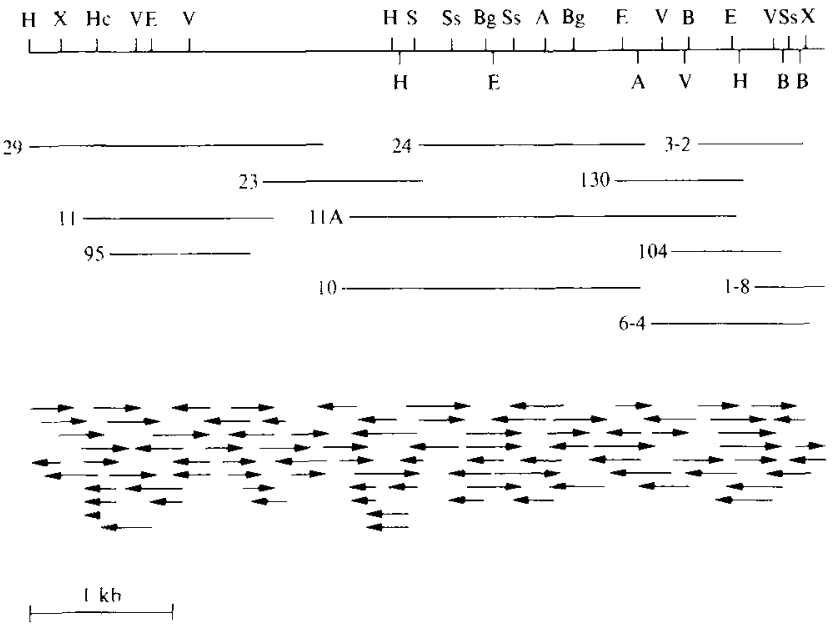

Fig. 1. Map of inserts from cDNA clones used for sequencing the BYDV NY-RPV isolate. The location of representative restriction sites within the genome is indicated. pRP cDNA clones used to generate the BYDV NY-RPV sequence are shown below the restriction map. The arrows indicate the length and direction of sequencing of these cDNA clones. A, Accl; B, BamHI; Bg, BglII; E, EcoRI; H, HindIII; Hc, HincII; S, SmaI; Ss, SstI; V, EcoRV; X, Xbal.

(Fig. 2), of which $100 \%$ was determined from sequencing of both strands and $74 \%$ was determined from more than one independently derived cDNA clone. The cDNA inserts in clones pRP11A, pRP23, pRP29, pRP95, pRP104, pRP3-2 and pRP1-8 were sequenced in their entirety, whereas only a partial sequence for the remaining clones shown in Fig. 1 was determined. Clones pRP29 and pRP1-8 represent the extreme $5^{\prime}$ and 3 ' ends of the NY-RPV sequence obtained, respectively; no other clones containing sequences coterminal with those obtained from either pRP29 or pRP1-8 were identified. Therefore, only pRP29 was used to determine the 5'-proximal 366 nucleotides and only pRP1-8 was used to determine the 3'-proximal 107 nucleotides.

Sequencing of more than one independently derived cDNA clone representing several regions of the genome identified six varying nucleotides (Fig. 2). Of these, half were $\mathrm{C}$ to $\mathrm{U}$ transitions and half were transversions, and three result in amino acid changes (Fig. 2); the other three changes, occurring in the 'wobble' position, would not result in any amino acid change. We do not have any indication whether the observed nucleotide differences are cloning artefacts or whether they represent true variability associated with the viral RNA population from which the cDNA library was constructed.

\section{Open reading frames $(O R F s)$}

A computer analysis of the NY-RPV nucleotide sequence identified six ORFs on the positive-sense strand capable of encoding polypeptides greater than 
$10 \mathrm{~K}$, as well as two on the negative-sense strand (Fig. 3). The amino acid sequences encoded by the six major positive-sense ORFs are indicated within the nucleotide sequence in Fig. 2 and are referred to as ORF1 to ORF6 in order of their proximity to the $5^{\prime}$ end of the sequence.

ORF1 begins at nucleotide 115 , which also begins the first AUG in the genome, and potentially encodes 256 amino acids of $M_{\mathrm{r}} 29263$. ORF2 begins with the second AUG in the genome at nucleotide 266 and potentially encodes a polypeptide of 654 amino acids $\left(M_{\mathrm{r}} 70575\right)$. Thus, ORF2 overlaps the $3^{\prime}$ end of ORF1 by 620 nucleotides. The first AUG of ORF3 begins at nucleotide 1813, which is 213 nucleotides downstream of the previous in-frame termination codon, and could encode a polypeptide of 633 amino acids $\left(M_{\mathrm{r}} 71785\right)$. The GDD consensus sequence for RNA-dependent RNA polymerase (Kamer \& Argos, 1984) has been identified within ORF3 (see below) and thus ORF3 encodes part of the NY-RPV viral replicase.

The coat protein of NY-RPV is encoded by ORF4 (Vincent et al., 1990). Beginning at nucleotide 3687, this gene is 612 bases in length, terminates with an amber termination codon and encodes a protein of 22190 . Located completely within ORF4, but in a different reading frame, is 9459 base ORF which is capable of encoding a protein of $M_{\mathrm{r}} 17211$ (ORF5). Recently the $M_{\mathrm{r}}$ of the VPg of a different RPV isolate has been determined to be $17 \mathrm{~K}$ (Murphy et al., 1989). As only ORF5 is capable of encoding a protein of $M_{\mathrm{r}} 17 \mathrm{~K}$, it is probable that it encodes the NY-RPV VPg. ORF6 is a 1197 nucleotide ORF beginning at nucleotide 4302, immediately after the coat protein gene termination codon and in the same reading frame. The first AUG of ORF6 does not occur until nucleotide 4572.

Western blot analysis of purified NY-RPV virions identified three proteins; Fig. 4 shows a typical analysis. Two smaller proteins were identified with estimated $M_{\mathrm{r}} \mathrm{s}$ of $19 \mathrm{~K}$ and $23 \mathrm{~K}$; the largest protein had an $M_{\mathrm{r}}$ of approximately $58 \mathrm{~K}$ to $63 \mathrm{~K}$, and often was not detectable (Fig. 4). Both the ability to detect the largest protein and its size were dependent on how often the purified virus preparation had been thawed and refrozen before analysis. When an NY-RPV preparation had been thawed and frozen five times, the $63 \mathrm{~K}$ protein could not be detected and a faint protein band with an apparent $M_{\mathrm{r}}$ of $58 \mathrm{~K}$ was identified instead (Fig. 4, lane 1). When this preparation was frozen and thawed once more, the $58 \mathrm{~K}$ band could no longer be detected.

Of the smaller proteins identified by Western blot analysis, the $23 \mathrm{~K}$ protein was predominant and migrated in SDS-PAGE as the authentic NY-RPV coat protein (Vincent et al., 1990); the band migrating as a $19 \mathrm{~K}$ protein was presumably the VPg. No separate ORF is apparent that would account for the largest protein observed by Western blot analysis of NY-RPV virions. However, readthrough of the coat protein gene termination codon could produce a protein of $M_{\mathrm{r}} 65 \mathrm{~K}$. The implication is that ORF4 and ORF6 together can encode a $65 \mathrm{~K}$ protein by readthrough of the ORF 4 stop codon, and that this protein is associated with the viral capsid. Similarly, readthrough of the PLRV coat protein gene termination codon recently has been shown (Bahner et al., 1990).

\section{Non-coding regions}

The NY-RPV genome contains 401 nucleotides that are not contained within the six major positive-sense coding regions. These non-coding regions are located at the extreme $5^{\prime}$ and $3^{\prime}$ ends of the genome, and $5^{\prime}$ to the coat protein gene (ORF4). We have no evidence that these $5^{\prime}$ and 3'-terminal sequences represent the actual termini of NY-RPV. We identified 114 nucleotides in the 5 -proximal non-coding region and 102 nucleotides in the 3 -proximal non-coding region. Computer analysis of the 3'-terminal non-coding region did not identify any potential tRNA-like structures. For PLRV and BWYV, luteoviruses with genomic organization similar to that of NY-RPV, there are 141, and between 146 and 197 3'-terminal nucleotides, respectively (Keese et al., 1990; Mayo et al., 1989; Veidt et al., 1988). A comparison between the 5 ' non-coding regions of these luteoviruses showed that although 114 nucleotides were identified at the $5^{\prime}$ end of NY-RPV, the length of this region is variable; isolates of PLRV have between 32 and 174 (Keese et al., 1990; Mayo et al., 1989), and BWYV has 31 nucleotides at the $5^{\prime}$ terminus (Veidt et al., 1988). However, for these luteoviruses, and also for Vic-PAV (Miller et al., 1988a), a consensus hexanucleotide sequence, AC/UAAAA/C, has been identified in these $5^{\prime}$ non-coding regions. This sequence is also found in southern bean mosaic virus (SMBV) (Keese et al., 1990; Wu et al., 1987).

Nearly half of all the non-coding nucleotides in NY-RPV are located between ORF3 and ORF4, i.e. $5^{\prime}$ to the coat protein gene. This region is A-U-rich, with $58 \%$ of the 185 nucleotides being $A$ or $U$ residues. Uridine forms $42 \%$ of the 113 nucleotides immediately upstream of the coat protein gene initiation codon, and $\mathrm{A}$ and $\mathrm{U}$ residues together make up $62 \%$ of the nucleotides in this region. A comparison of similar regions from other luteoviruses indicates that there is a core region consisting of 113 nucleotides upstream of the coat protein gene initiation codon (Fig. 5). This core region contains the identifying features of subgenomic promoters defined for brome mosaic virus (BMV) (Marsh et al., 1988). There is extensive nucleotide identity between the core regions of NY-RPV, BWYV 
ORF1

121

241

ORF 2

361

ORF1

481

ORF 1

ORF 2

601

721

ORF 1

841

ORF 1

ORF 2

961

(1)

1081

ORF 2

1201

ORF 2

1321

ORF 2

1441
ORF2

1561

ORF 2

ORF

1681

ORF 2

ORF 3

1801

ORF 2

ORF 3

1921

ORF2
ORF 3

2041

ORF2

ORF 3

2161

ORF 2

ORF 3

2281

ORF 3

2401

ORF 3

2521

ORF 3

2641

ORF3

2761

ORF 3

CGGCUCCAAAGCUUAAGCUCACUGUUCCCGCGCUGUUUCGACGUUCCAGCUCACAGCCCAUAAAACCCCCUCCUCCCGCUCUAGCGAUCAACUCGCGUGUUCGUUAUUAACGCAUGUUC

AUCGCCCAACCUUGCGGGCGAGUUCUUGUGUUCGACGUCGCCUCCCACACGCCAUCGUUCUUCACUCGUUAUAGUGUUGAACUCUCGCUCCGUGCUCUAGAUCCACUCUUCACGCGAGCA $\begin{array}{llllllllllllllllllllllllllllllllllllllllllll}I & A & Q & P & C & G & R & V & L & V & F & D & V & A & S & H & T & P & S & F & F & T & R & Y & S & V & E & L & S & L & R & A & L & D & P & L & F & T & R & A\end{array}$

GUAACAGAUUUCCGAUACACCCAAAAUGAAAUCGAUUUAUUUUGUGUGUCUCUUGGCUUUCUGUUGCCAAUUCUCCUCACAGGAGAAUCUUACUCUUGGCGCGGUCACCUUAACCUCCCC

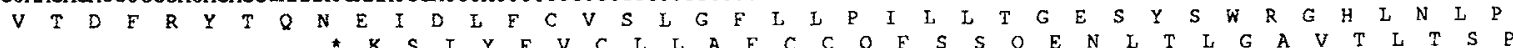

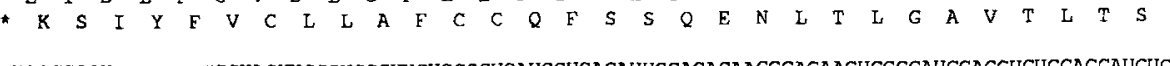
CUUUCUUACACCGAACUACUUGUUCGAUGGGGGCUCGCAGUGGGGUACUUCCCUGCCUUCUCCGCUGAUGGUGACAUUCGACAGAACCCAGAACUCCGCAUCGACCUGUCCACCAUGUCA

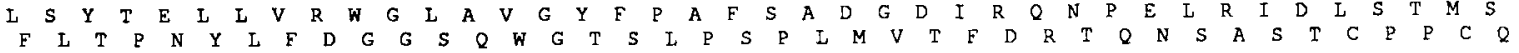
ACCCGCUCUUUCUACGAGCAGUUCCUACUCAGAUAUAAUACAAGUGGGUUGGCAAAAGCUAUCGUUGGACAGCAAGAGUGCUUUCAAAGCGGCAUGGAGUCUUUUAAAAGAUUCCUGCAC

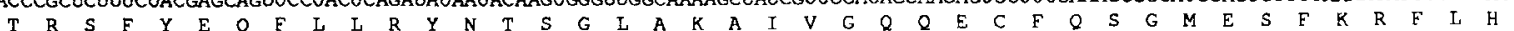

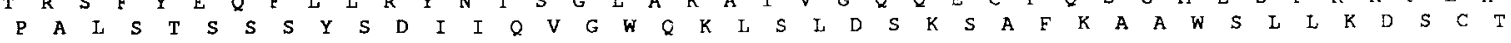
UACCGCCUCACGUGCUUUGAAAGCUGCCUUCCACGACCUCGUUGGGAAAGCCCUUUGGCUCCUGGUCCUUAUCUGGACAGGGCUUUUGAGGCAACUCUUCUCGGCCGUAUGGUCGGCCAU

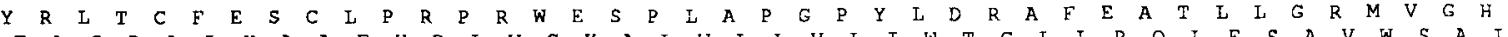

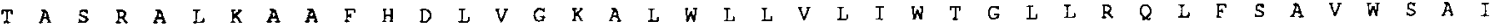

AACCAACUACUCUUUACCGGUUUGUCUUCUGAUAUCACUAGGUAUUAUAACGAGUUGGUUGUGGAAGGCGUGCCGGUGGCUUUUUGGGACGCUGCCGGCAUUACUUUUGCAUCACGCUGGU

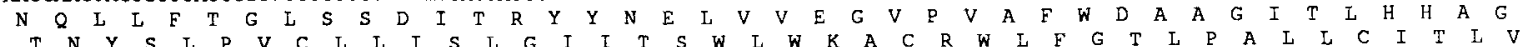
GAAGAAUAUUUUCCGAAUUCUUACAUUCAAAAGAUUCUUCAAUGAGAAAACUGUCUCAGGUUAUGACAGUUAUUCGAUUCCCAGCACCCCUCCGAAGCGGAGCGUCAUCAUGAUGCGACG E E $E$ Y $F$ P

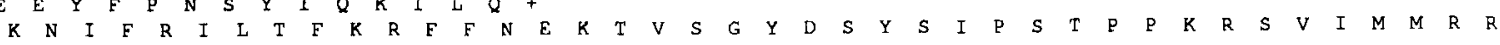

(C)

CCAAAAUAAGGAGCACAUUGGAUAUGCGAACUGUAUUCGUCUUUUCGACGGACGGAACGCAAUUGUCACAGUUGCUCACAAUAUAGAGGAGGGUUGCUCUUUCUAUUCCUCACGGACUAG

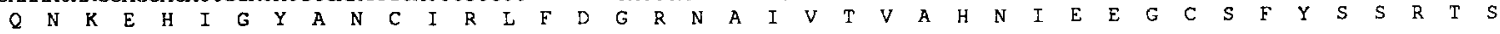
(P)

UGGUUCCAUCCCAAUCACUGAAUUUCGCGUUAUCUUUGAAAGCAAAACCAUGGAUAUCGCCAUUCUGGUGGGACCCAUCAAUUGGGAAUCGAUUCUCGGAUGCAAAGGAGUCCACUUUAC

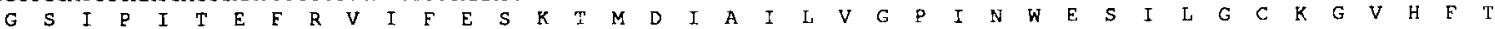
CACCGCUGACCGCUUAGCAGAAUGUCCAGCGGCUCUUUAUCUUCUUGAUAGCGAUGGUCAGUGGCGCUCAAACUCGGCGAAGAUUUGUGGUCAUUUCGACAACUUCGCCCAAGUCUUGUC

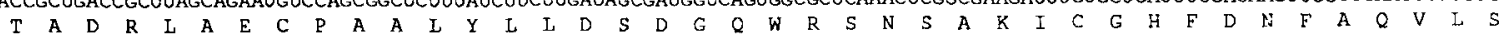

(U)

CAACACUAAAGUCGGUCACUCCGGUGCCGGUUACUUCUAUGGCAAGACUCUCGUUGGCCUCCAUAAAGGCCAUCCUGGCAAAGAUUUCAAUUUUAAUCUGAUGGCUCCUUUGCCAGGAAU $\begin{array}{lllllllllllllllllllllllllllllllllllllllllll}N & T & K & V & G & H & S & G & A & G & Y & F & Y & G & K & T & L & V & G & L & H & K & G & H & P & G & K & D & F & N & F & N & L & M & A & P & L & P & G & I\end{array}$ ACCCGGCUUGACCUCCCCGCAGUACGUCGUGGAAUCCGACCCCCCCCAGGGAUUGGUUUUCCCCGAAGAAGUCACUGAAUCAAUCGAAGCCGCGAUUAAGGAGGCAACAAUGUACAAGAA $\begin{array}{lllllllllllllllllllllllllllllllllllllllll}P & G & L & T & S & P & Q & Y & V & V & E & S & D & P & P & Q & G & I & V & F & P & E & E & V & T & E & S & I & E & A & A & I & K & E & A & T & M & Y & K & N & N\end{array}$ UGUCUUCGCCAAUAGAGGACGUGGCGCCUUUAAAUCUAAAAGCGGCAUCAACUGGGAAGACAUCGAAGACGAGUCGGGAAACGGGAAGGCGGCGGCGUCCGCCGUAACAAACGCAGCAGC

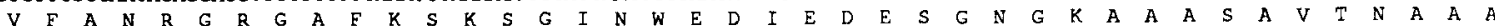

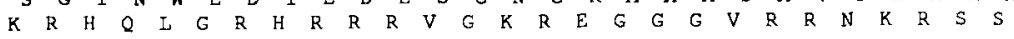

GGCAAACAAGGUUAUCGCCACCCCCGGCGUCGCCAAGUCCCAAAAGAAGACUGCUGUUCCUUCUUCGCCGAAGGCACCGCAGCCCCCCGCUGCAUCCCAACCCACCAGCACAACUGGGAG

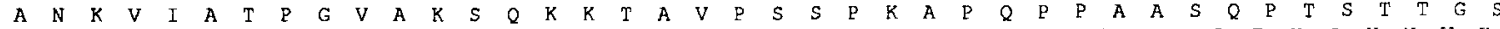

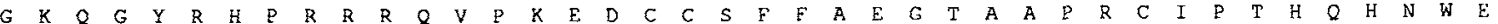

CAGAACUCCUAUAUGUCCAAUUGCUACUGCAACAUCCCCGGACACGGCUGUGCCUACUGGACCAUCGCAGCAAGAAAUCAUGAACAACAUAAUGAAUCUGCUGGUCCAGAGGAUCGAUAJ

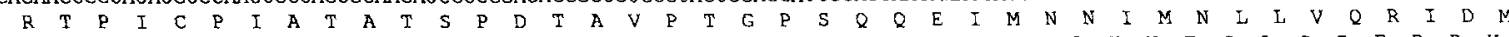

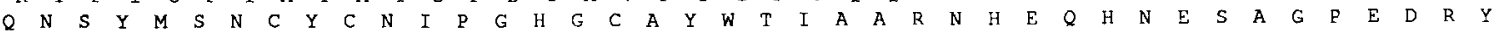

GUCGAAGAUCGAGAAAUCGAUAGUGGACCAAGUCGCGAAUCAAGCUCUGAAGAAACCACGCGGCAAGCGUGGCUCAAAGAAACGGCCCGCAGCUGGCAAAAGUUCUUCGCCGACAUCUAC

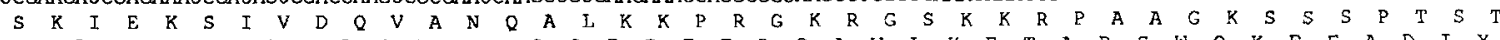

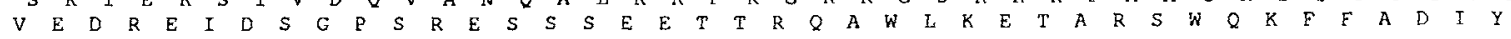

ACCUGGGACGUAUCAACAUCCAAACAAGAAGUCCCAGGCUUCGAACAGGUUGGGAAAUUCUCCCCCCAGUACUACCCGCGCCCCCGCAGCGAAUCAGAAUGGGGGAGAAAACUCUGUGCC

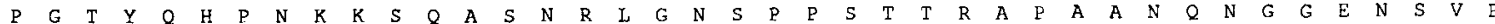

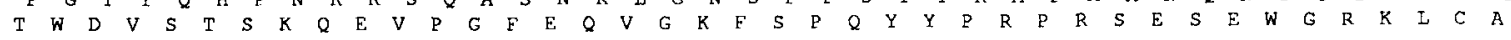

GAACACCCUCUCUUGGGUGAGAAAACUGCCGGUUUCGGGUGGCCCCAAGUCGGGGCAUCCGCUGAACUGACAUCUCUUCGGCUGCAAGCAGCUCGCUGGCUCGAACGUUCCGAGUCAGCU

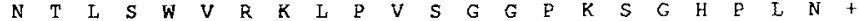

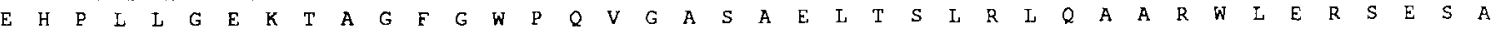

$\langle\mathrm{U}\rangle$

AAAAUUCCAUCAGAUGCUGCUAGAGAGAACGUGAUCAAUCGCACCGUACAGGCGUACUCGAAUUGCAAAACCAAUACGCCGAGGUGCACACGAGGUGAGUUAAGUUGGGAAACGUUCAAA

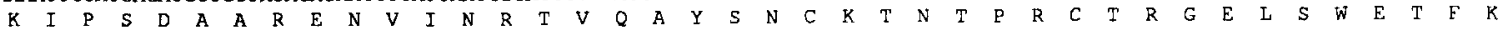
AUUGACUUCCUUGAAGCCAUCAAAUCGCUCCAACUUGACGCUGGCGUCGGAUUACCGAUGAUCACUGCGGGGCUCCCCACCCAUCGUGGGUGGGUUGAAGACCCAGAUCGUUUGCCAGUG

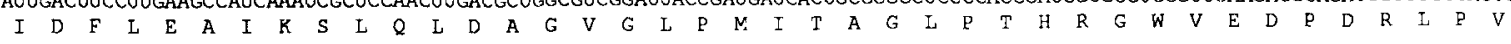

UUAGCUCAGCUUACCUUUGACCGCCUACUUACGAUGUCAAAGGCAAGCUUGGAAGCUCGCUCCCCGGAGCAACUCGUGAAGGAGAAUCUCUGUGAUCCUAUUCGUCUCUUUGUAAAACAA

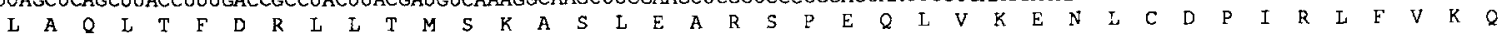

(U) GAGCCACAUAAACAGAGUAAGCUUGAUGAGGGACGUUACCGCCUCAUCAUGUCAGUCUCCUUGAUUGAUCAAUUGGUAGCCCGGGUUUUGUUUCAAAGACAAAACAAAUCCGAAAUCGCG

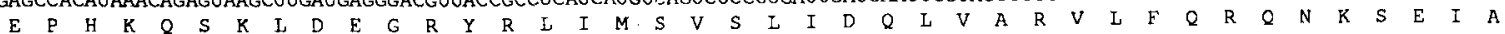
UUGUGGAGCGCGAUUCCGUCAAAACCCGGUUUUGGAUUAUCCACCGAGGACCAAGUUUCAAAAUUCAUGGAUGUCCUCGCCGGGAACGUUGGUGCGUCUCCCGAAGAGGUCUGCGACAAU

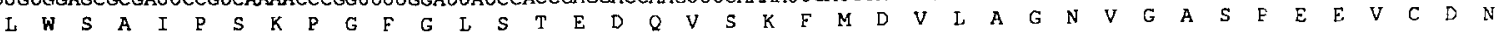


(C)

2881

$\mathrm{ORF} 3$

3001

ORF 3

3127

ORF 3

3241

ORF 3

3361

ORF 3

3481

ORF 3

3601

ORF

ORF 5

3721

ORF 4

ORF 5

3841

$\mathrm{ORF}$

ORF

3961

ORF4

ORF 5

4081

$\mathrm{ORF}$

ORF 5

4201

ORF 4

4321

ORF 6

4441

ORF 6

4561

ORF'

4681

ORF 6

4801

ORF 6

4921

ORE 6

$504]$

ORF 6

5161

ORF 6

5281

ORF 6

5401

ORF 6

5521

UGGCGCGACCUGUUGGUUCCUACGGACUGUUCCGGUUUUGACUGGUCAGUCUCCGAUUGGAUGCUCGCGGAUGACAUGGAGGUGAGAAAUCGCCUAACAAUCGACUGCAACGAGCUCACC $\begin{array}{lllllllllllllllllllllllllllllllllllllllll}W & R & D & L & L & V & P & T & D & C & S & G & F & D & W & S & V & S & D & W & M & I & A & D & D & M & E & V & R & N & R & L & T & I & D & C & N & E & L & T & \end{array}$

(R)

GACACCUUAGGGCUGUUUGGCUUCAGGGCAUAUCAAACUCGGUUUUAUGCCUGUCGGAUGGAACCAUGUUAGCUCAACGAGUACCCGGCGUGCAGAAAAGUGGUUCGUACAACACUUCC

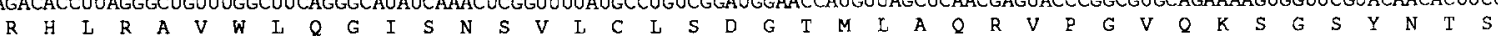

UCCACAAACUCACGCGUUCGGGUCAUGGCUGCCUAUCACUGUGGCGCCUCGUGGGCUAUCGCUAUGGGUGAUGAUGCCCUGGAAGCUCCAGACACAGACUUGUCAAAAUAUAAAGAUCUG $\begin{array}{llllllllllllllllllllllllllllllllllllllllll}S & T & N & S & R & V & R & V & M & A & A & Y & H & C & G & A & S & W & A & I & A & M & G & D & D & A & L & E & A & P & D & I & D & I & S & K & Y & K & D & L\end{array}$

GGCUUCAAAGUCGAGGUUAGCGGAGAGUUGGAAUUCUGCUCGCGCAUUUUCAAGACCCCUAACCUCGCCAUUCCGGUUAAUGAAAACAAAAUGUUGUACCGCUUGAUCCACGGGUACAAU

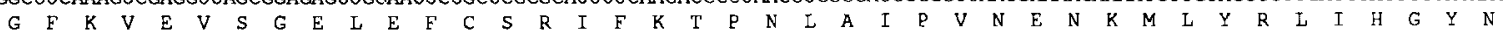

CCGGAAUGUGGCAAUUUUGAGGUGGUUCAAAACUAUCUCAAUGCAGCUGUCUCAGUGCUGCAUGAGCUCCGACAUGAUCGGGAGCUUUGCCUAAAACUUCAGGAGUGGUUGAUUUCUGAC

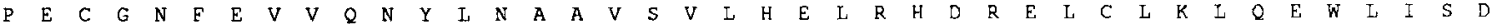

GUCACCACAAAACUAAACUGAGCACAAAACUAGCCGGACAAACGUAAGUUGCAAGUGCCGGAAGUCAAGUCUUACACACAAGCCCAACAUUGAUUUUCAUUUGUUAGCGGGAUUUGCCCU $V$ T T K L N

UGGAUUUAUAUCCUCAAUCCCCAUUUCAGUUGUUGGUGUUUAUCUAGUCUACCUUAAGGUUUCGACCCACAUUCGUGAAAUUGUUAAUGAGUACGGUCGUCCUUAGAUCCAAUGGCAAUG * S T V V L R S N G N

GUUCGCGCAGACGCAGACAGAGAGUCGCUCGGCGAAGGCCUGCUGCAAGAACGCAGCCAGUGGUUGUGGUCGCUUCCAACGGCCCAGCCAGGCGCGGAAGACGCCGACGACCAGUUGGUC $\begin{array}{llllllllllllllllllllllllllllllllllllllllllll}G & S & R & R & R & R & Q & R & V & A & R & R & R & P & A & A & R & T & Q & P & V & V & V & V & A & S & N & G & P & A & R & R & G & R & R & R & R & P & V & G\end{array}$

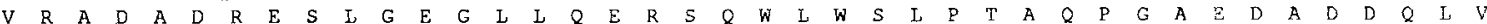

CUCGGCGAGGAAGAACUCCAAGAUCUGGAGGAGGAAGCCGUGGCGAGACAUUCGUUUUCUCAAAGGAUUCACUCGCGGGCAACUCCUCUGGAAGUAUCACCUUCGGGCCGUCUCUAUCAG

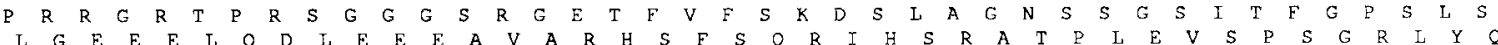
AGUAUCCGGCAUUCCAGAAUGGAGUACUCAAGGCCUACCAUGAAUAUAAGAUCACAAAUUGUGUCUUACAGUUCGUCAGCGAGGCCUCUUCCACAGCAGCCGGCUCCAUCUCUUACGAGU

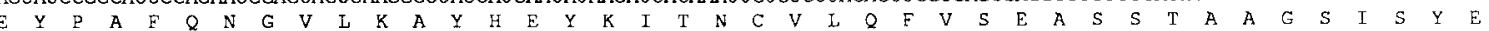

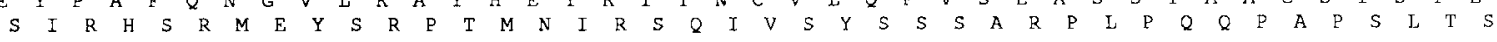
UGGACCCCCAUUGCAAAGCAUCUUCACUCGCAUCAACAAUCAAUAAGUUCACAAUCACCAAAACUGGUGCGCGGAGCUUCCCAGCGAAGAUGGAUCAACGGGUUAGAGUGGCACCCCUCAG

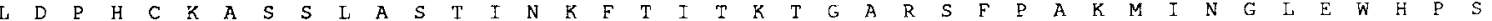

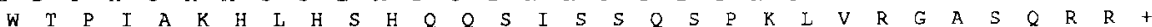

AUGAGGAUCAGUUCCGAAUUCUUUACAAAGGGAACGGUGCUUCUUCUGUGGCUGGGUCUUUUAAGAUCACUCUUAGAGUCCAGCUACAAAACCCAAAAUAGGUAGACGCGGAACCCGGUC

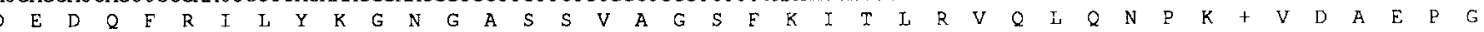
CCAGUCCAGGACCAUCACCCGACCCCCCCCCCCACCUUCACCGUCCCCAGAACCUGCUCCUGCUAAGGAGGAACGAUUUAUCGUCUAUUCAGGGGUGGCUCACACAAUCAUCAGUGCCC

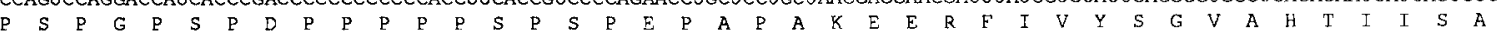

AAAGCACUGAUGAUUCUAUCAUAGUUAGAGAUAUCCCAGACCAACGGUUUAGAUACGUUGAGAACGAAAACUUCUACUGGUUUCAGAUAGCUGCUCAAUGGUACUCAAAUACGAACACAA

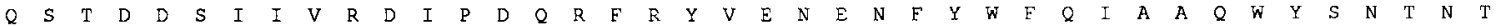

AAGCAGUUCCGAUGUUUGUCUUUCCAGUCCCCAUCGGGGAAUGGUCAGUCGAAAUAUCAACUGAGGGAUAUCAAGCUACGUCUAGUACCACGGAUCCUAACAAGGGCCGCAUUGACGGGC $\begin{array}{llllllllllllllllllllllllllllllllllllllllll}K & A & V & P & M & F & V & F & P & V & P & I & G & E & W & S & V & E & I & S & T & E & G & X & Q & A & T & S & S & T & T & D & P & N & K & G & R & I & D & G\end{array}$

UCAUAGCUUAUGAUAACUCAAGUGAAGGGUGGAAUAUCGGGGCUGGAAGUAACGUUACCAUCACGAACAACAAAGCUGACAAUAGUUGGAAAUACGGUCAUCCUGAUCUGGAAAUCAACU

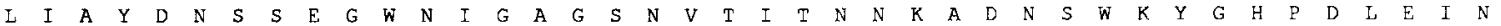

(A) CUUGUCACUUCAACCAGAAUCAAGUUUUGGAGAAAGAUGGUAUUAUUUCUUUCCAUGUCAAGGCUACAGAGAAGGAAGCUAAUUUCUUCUUAGUCGCCCCUCCAGUCCAGAAAACCUCGA $\begin{array}{llllllllllllllllllllllllllllllllllllllllllllll}S & C & H & F & N & G & N & Q & V & L & E & K & D & G & I & I & S & F & H & V & K & A & T & E & K & E & A & N & F & F & L & V & A & ? & P & V & Q & K & T & S\end{array}$ (K)

AGUAUAACUACGCUGUCUCAUAUGGAGCGUGGACGGACAGAGACAUGGAAUUCGGAUUGAUUACAGUCACGUUAGAUGAGAAACGUGGCUCGGGUUCCCCCACUCGCAAAAGCUUACGCG

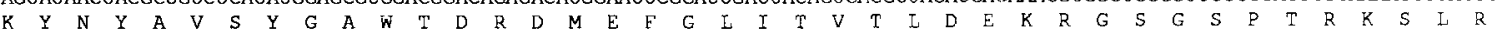

CUGGACACGCGGGAGUAACGACCACCACUGAUUUGGUGGCGUUACCGGAGAUGGAAAACUCCGGUAUAGAAACAUCUGAAACCCCGUCAGCUCCGGUCACCAGCUCAAAAGCACCGUUGC

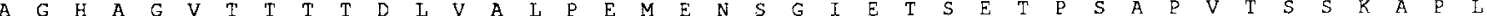

CAACUGUCUCGGAUUCGGAGUCAGAAGACGAUCCGUUAUCAGCCGCACCAGAUGUAGGCUUUGGCGGAACUCGUCUUUUGAUUGAUACUGAUAUCAAAACAAUACCCGAUCCGGACGUUG

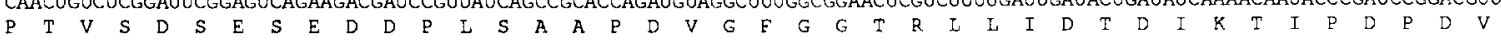

CUGAUGCCUUCGUGAACAGCGCUCACGUGGGUGUGGAUCCAUGGGCAGAAGUUCGAGCGUUUAAGAGAGCUCAGCGUCCUCCCCGUGGACCGUCAUCGGUUGCUAGCAGCAGCCUCUCUG $\begin{array}{lllllllllllllllllllllllllllllllllllllllllllll}A & D & A & F & V & N & S & A & H & V & G & V & D & P & W & A & E & V & R & A & F & R & R & A & Q & R & P & P & R & G & P & S & S & V & A & S & S & S & L & S\end{array}$

GAGGUUCGCUGCGUGGAAGCCUCAGACCGAAAACUGAGGAUCCGAAAGAUUCAUCCAAAUCUAAAUCUAGAAAAUGGUCUCUCGGAAGUCUGCGAUGACUAUAAACUAAGGCGAAGGAAA $\begin{array}{lllllllllllllllllllllllllllllllllll}G & G & S & L & R & G & S & L & R & P & K & T & E & D & P & K & D & S & S & K & S & K & S & R & K & W & S & L & G & S & I & R & +\end{array}$

AUGCCUUAAAUCACCCGUCAAGCGACGUUAAAAGGAGGAUUAAGACCCUCCCCCCUUGCUGGUUCAACAACCUUGUUCCO

Fig. 2. Nucleotide sequence (RNA form) of the BYDV NY-RPV isolate. The initiation codons for the six major positive-sense ORFs are indicated by an asterisk and the termination codons by + . The deduced amino acid sequence is indicated below the nucleotide sequence. Nucleotides which varied when identified from the sequencing of different clones are indicated in parentheses above the nucleotide sequence; associated changes in the deduced amino acid sequence are indicated in parentheses below the amino acid sequence. 


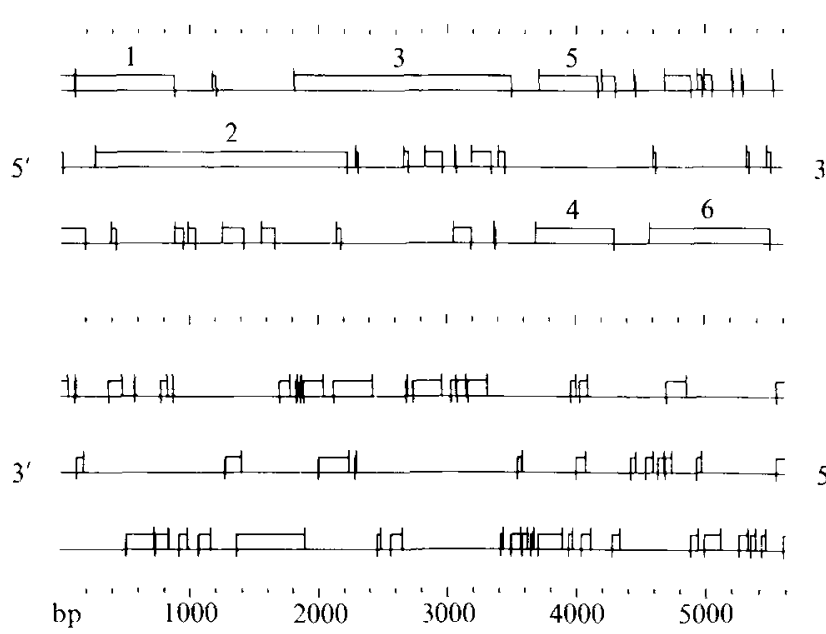

Fig. 3. ORFs determined from the positive- and negative-sense orientation of the BYDV NY-RPV nucleotide sequence. Vertical ticks above a line indicate the location of initiation codons; those below a line indicate the location of termination codons. The open boxes represent ORFs and the six major positive-sense ORFs are numbered according to their proximity to the $S^{\prime}$ end of the genome. All ORFs are represented as beginning with an AUG initiation codon.

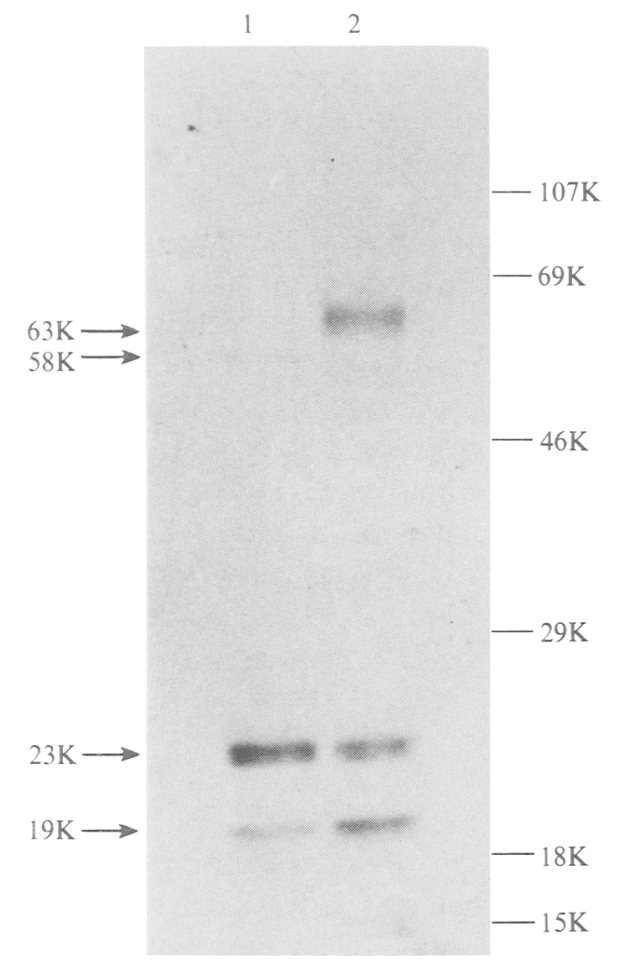

Fig. 4. Immunological detection of the virion-associated proteins from the BYDV NY-RPV isolate. Purified NY-RPV virions were dissociated and electrophoresed on a $10 \%$ polyacrylamide gel. After electroblotting onto nitrocellulose, virus-associated proteins were detected immunologically using a polyclonal anti-NY-RPV primary antiserum and an alkaline phosphatase-conjugated secondary antiserum. Sizes of proteins identified from purified NY-RPV preparations are shown to the left, those of protein size standards to the right. Lane 1, purified NY-RPV thawed and frozen five times; lane 2, purified NY-RPV thawed only once.

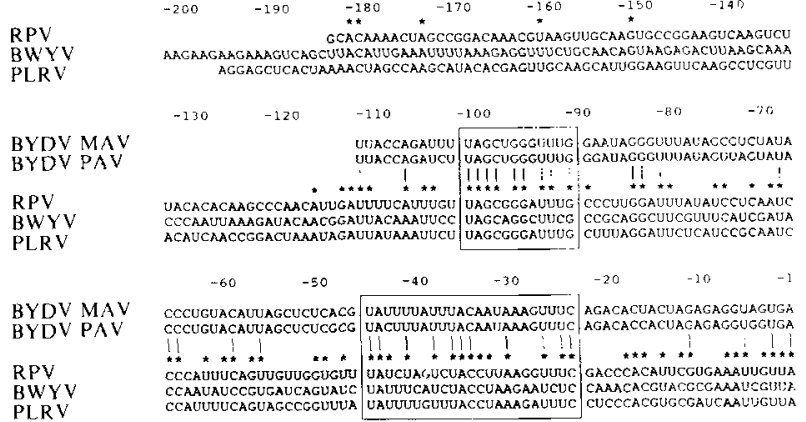

Fig. 5. Comparison of luteovirus non-coding regions located 5 to the coat protein coding region. Asterisks represent nucleotides identical in the sequences of BYDV NY-RPV isolate, BWYV and PLRV. A vertical bar represents nucleotides identical in all the luteoviruses examined. Numbering is in a direction $5^{\prime}$ to the putative coat protein gene initiation codon. Boxed regions represent regions conserved in luteoviruses. RPV, BYDV NY-RPV isolate; BWYV, BWYV FLI (Veidt et al., 1988); PLRV (Mayo et al. 1989); BYDV MAV (P. P. Ueng \& R. M. Lister, unpublished results); BYDV PAV (Miller $e t$ al., $1988 a$; P. P. Ueng \& R. M. Lister, unpublished results).

and PLRV, and two highly conserved regions found in all luteoviruses sequenced. The high degree of nucleotide conservation, and the presence of A-U-rich tracts and AUCA and AAGA sequence blocks similar to those of BMV subgenomic promoter sequences, suggest that this region may be involved in the formation of subgenomic dsRNAs and/or the regulation of specific protein messages. In fact, the transcriptional start site of a $2.6 \mathrm{~kb}$ subgenomic RNA from PLRV has recently been mapped to $34 \mathrm{bp} 5^{\prime}$ to the coat protein gene translation initiation codon (Tacke et al., 1990), which corresponds to the larger of the two conserved regions. The size of the dsR NAs identified in oat plants infected with NY-RPV (Gildow et al., 1983) is consistent with this region having a role in subgenomic RNA formation.

\section{Comparison with other luteoviruses}

The NY-RPV sequence is the second BYDV genome sequence to be reported. The genome of a BYDV PAV serotype from Victoria, Australia, Vic-PAV, has been determined to consist of 5677 nucleotides (Miller et al., $1988 a$ ) and encodes six positive-sense ORFs. The genome organizations of NY-RPV and Vic-PAV show significant differences (Fig. 6). Although the RNAs of both contain approximately 5600 nucleotides, there is no similarity in genome organization and little sequence similarity in regions of the genomes $5^{\prime}$ to the coat protein genes. The coat protein gene itself is located 3'-proximal in NY-RPV, whereas it is near the centre of the genome of Vic-PAV. 

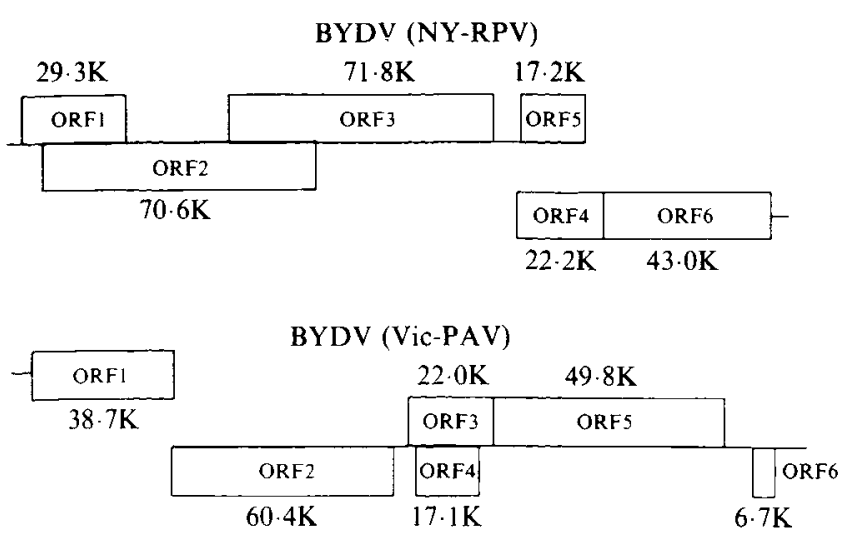

Fig. 6. Genome organization of BYDV NY-RPV and Vic-PAV isolates. The location of major ORFs of NY-RPV and Vic-PAV (Miller et al., 1988a) and the reading frames in which they occur are represented by boxes. The sizes of the polypeptides potentially encoded by each ORF are indicated.

However, similarities between Vic-PAV and NY-RPV are found in the $22 \mathrm{~K}$ ORFs, in the internal $17 \mathrm{~K}$ ORFs and in the immediately 3 '-proximal ORFs (i.e. NY-RPV ORF6 and Vic-PAV ORF5). The 22K ORFs are the coat protein genes and contain the presumptive $17 \mathrm{~K} \mathrm{VPg}$ gene as an internal ORF. This organization has been found in all luteoviruses examined to date (Vincent et al., 1990). Nucleotide and deduced amino acid sequence identities between NY-RPV and Vic-PAV ORFs determined using Microgenie sequence alignment software were $55.2 \%$ and $48.8 \%$ for the coat protein coding region, $51.7 \%$ and $34.7 \%$ for the $\mathrm{VPg}$ coding region, and $44.6 \%$ and $32.2 \%$ for the full ORFs located immediately $3^{\prime}$ to the coat protein coding region. The ORF 3'-proximal to the NY-RPV coat protein ORF is proposed to be expressed by readthrough of the coat protein gene termination codon, as with a similar ORF of Vic-PAV and, indeed, with luteoviruses in general. A conserved 14 nucleotide sequence surrounding the luteovirus $22 \mathrm{~K}$ coat protein gene termination codon has been identified previously, and a role for this sequence in the expression of the readthrough ORF postulated (Smith \& Harris, 1990; Veidt et al., 1988). An examination of this region from NY-RPV indicates that this conserved region is actually the 12 nucleotide sequence AAAUAGGUAGAC, with the coat protein gene termination codon indicated by the underlined nucleotides.

The genome organization of NY-RPV, although it differs from that of Vic-PAV, is very similar to that reported for both BWYV (Veidt et al., 1988) and PLRV (Keese et al., 1990; Mayo et al., 1989). Furthermore, the sizes of the putative protein products encoded by each ORF of the latter viruses are similar to those encoded by the ORFs of NY-RPV.
There is considerable interest in evolutionary relationships between viruses based on amino acid sequence motifs associated with the RNA-dependent RNA polymerases (viral replicases) (Habili \& Symons, 1989; Kamer \& Argos, 1984; Poch et al., 1989) and nucleic acid helicases (Gorbalenya et al., 1988; Gorbalenya \& Koonin, 1989; Habili \& Symons, 1989; Hodgman, $1988 a, b)$. These relationships are based both on sequence similarity within motifs and on the distance separating motifs. Using such a classification scheme, positive-sense RNA plant viruses have been placed into three supergroups, with the luteoviruses in supergroup B (Habili \& Symons, 1989).

The Vic-PAV replicase shows substantial similarity to the replicase of carnation mottle virus (Miller et al., 1988a), and the replicases of BWYV and PLRV show homology to that of SBMV (Mayo et al., 1989; Veidt et al., 1988), all members of supergroup B. A BESTFIT comparative analysis (Devereux et al., 1984) of the amino acid sequence deduced from NY-RPV ORF3 with that of the putative replicase-encoding region of SBMV (Wu et al., 1987) has identified a region containing the GDD amino acid motif for RNA-dependent RNA polymerases (Kamer \& Argos, 1984). This region is also similar to regions of both PLRV and BWYV (Fig. 7). The four amino acid sequence motifs of supergroup B positive-sense RNA viral replicases (Habili \& Symons, 1989) (VPTDCSGFDWS-53GVQKSGSYNTSSTNSRV-11-WAIAMGDDALEA22-FCSRIF) were also identified within this region (Fig. 7), although the last motif was not represented in SBMV. The NY-RPV replicase motifs showed greater similarity in both sequence and gap distances to comparable regions in BWYV and PLRV, than to those in Vic-PAV.

Seven amino acid sequence motifs have been identified for proteins that have helicase functions (Habili \& Symons, 1989; Hodgman, 1988a, $b$ ), and have been used to evaluate the taxonomic relationships between luteoviruses and other positive-sense RNA plant viruses (Habili \& Symons, 1989). The NY-RPV sequence contains three of the seven helicase motifs. These regions resemble the ' $A$ ' and ' $B$ ' sites of the catalytic site of nucleoside triphosphate-utilizing enzymes (Gorbalenya et al., 1988; Gorbalenya \& Koonin, 1989) and supergroup B motif VI (Habili \& Symons, 1989). The potential ' $A$ ' and ' $B$ ' sites are located in ORF2 (356-AGYFYGKT-41-LVFPEE-240), and motif VI has been identified in ORF3 (351-QSKLDEGRYRLIMSVS-267). The NY-RPV helicase motifs were compared with those from other luteoviruses (Habili \& Symons, 1989). Motif VI has been found in all luteoviruses, but although all other luteoviruses contain motif IV, no such region was identified in NY-RPV. Regions comparable to the ' $A$ ' and ' $B$ ' motifs were not identified in PLRV or Vic-PAV, 


\begin{tabular}{|c|c|c|c|c|}
\hline RPV & 326 & ARSPEQLVKENLCDP IRLFVKOEPHK & QSKLDEGRYRLIMSVS & LIDQLV \\
\hline PLRV & 308 & DMSAEE LVQEGLCDP I RLFVKREPHK & QSKLDEGRYRLIMSVS & LVDOLV \\
\hline & & 1111111111111111 & $1: 111111111111111$ & 111111 \\
\hline BWYV & 276 & DMGPEELVRNGLCDP IR FVKGEP HK & QAKLDEGRYRLIMSVS & LVDQLV \\
\hline & & & 11111 & 11 \\
\hline RPV & 326 & $\begin{array}{l}\text { ARSPEQLVKENLCDP IRLFVKQEP HK } \\
|||||||||:|||||||\end{array}$ & $\begin{array}{l}\text { QSKLDEGRYRLIMSVS } \\
|||||||||||l|\end{array}$ & $\begin{array}{l}\text { LIDQLV } \\
:: 1] 11\end{array}$ \\
\hline SBMV & 600 & ALSP TEMVEMGLCDPVELFVKOEF HP & SRKLKEFRYRLISSUS & IVDQLV \\
\hline Vic-PAV & 121 & 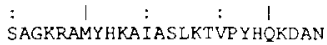 & $\begin{array}{c}1 \quad: \quad: \\
\text { VQAF LKKEKHWMTKDI }\end{array}$ & $\begin{array}{l}\text { : } \\
\text { APRLIC }\end{array}$ \\
\hline
\end{tabular}

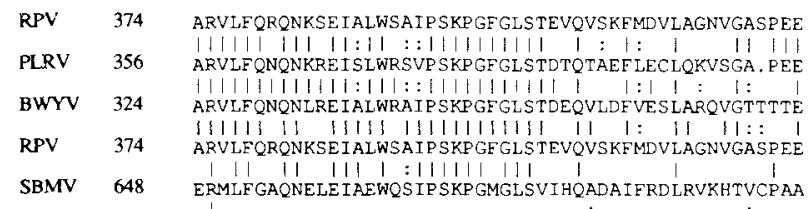

VIC-PAV 169 PRSKRYNIILGTRLKFNEKKIMHAIDSVFGSPTVLSGYDNFKOGRIIAKK

\begin{tabular}{|c|c|c|c|c|}
\hline RPV & 424 & $\mathrm{CDN}$ & VRTDCSGFDWS & VSDWMLADOMEVRNRLT IDCNELTRHLR \\
\hline & & & $|1| 1|1| 1|1| \mid$ & $|1||| 1|1| 1|||| 1$ \\
\hline PLRV & 406 & LCANHKEYT & RPTDCSGFDWS & VRYMMLEDDMEVRNRLT FNNTQLT KRLR \\
\hline BWYV & 374 & \begin{tabular}{lll|l}
$\vdots$ & 1 & 1 \\
VVANWKNYL
\end{tabular} & $\begin{array}{c}\text { IIIIIIIIIIIII } \\
\text { TPTDCSGFDWS }\end{array}$ & 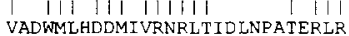 \\
\hline & & $11:$ & 1111111111 & $1: 1111|111| 1111111111: 11$ \\
\hline RPV & 424 & VCDNWRDLL & $\begin{array}{l}\text { VPTDCSGFDWS } \\
: i||||||\end{array}$ & 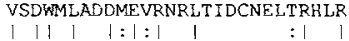 \\
\hline SBMV & 698 & & EADISGFDWS & VODWELWADVEMRIVLGSFPPMMARAAR \\
\hline Vic-PAV & 219 & WQKFACI & $\begin{array}{c}|1| \mid \\
\text { IGVDASRFDQH }\end{array}$ & \begin{tabular}{ll|l} 
& 1 & $\vdots$ \\
VSEQALKWEHGIYNGIFGDSEMALALEH
\end{tabular} \\
\hline
\end{tabular}

\begin{tabular}{|c|c|c|c|c|c|}
\hline & & & ‡ & * & \\
\hline RPV & 472 & SDGTMLAQRVP & GVOKSGSYNTSS & TNSRV & RUMAAY \\
\hline PI.RV & 454 & $\begin{array}{l}1|1| 1|1| 1|1| 1|1| 1|=| 1|||1| \\
\text { AAWLKCIGNSVLCLSDGTLLAQTVP }\end{array}$ & $\begin{array}{l}1 \text { H I I ! I I I I I I I } \\
\text { GVOKSGSYNTSS }\end{array}$ & & 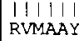 \\
\hline & & $: 11:|1| 1|1| 111111111$ & $1\|111\| 1 \| 111$ & 1111 & $11111:$ \\
\hline BWYV & 422 & SCWLRCISNSVLCISDGTLLAQIHP & GVQKSGSYNTSS & SSNSR I & RVMAAF \\
\hline RPV & 472 & 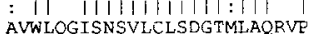 & GVOKSGSYNTSS & & RVMAAY \\
\hline SBMV & 736 & 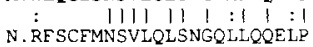 & 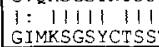 & $\begin{array}{l}\text { 1111: } \\
\text { STNSRI }\end{array}$ & $\begin{array}{l}1: 1 \\
\text { RCLMAE }\end{array}$ \\
\hline Vic-PAV & 267 & $\begin{array}{ccc}1 & \vdots & \vdots\end{array}$ & GDIN & 1CGMM & $\begin{array}{c}\vdots \\
\dot{H} A Y L K K \\
\end{array}$ \\
\hline
\end{tabular}

\begin{tabular}{|c|c|c|c|c|c|}
\hline & & & & & \\
\hline RPV & 520 & Co & AIAMGDD & .PDTDLSKYKDLGFKVEVSGELE & FCSRIF \\
\hline $\mathbf{R}$ & & & & 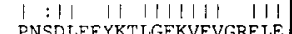 & 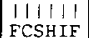 \\
\hline & & & & $: 11111111111$ & 111 \\
\hline 3 WrV & 470 & HTGAI & WA & . NP AD LAAYKKLGEKVEVSGQLE & FCSHIF \\
\hline$P$ & & & & 1111 & 111 \\
\hline P & 520 & GAS & & $\begin{array}{c}\text { PDTDLSKYKDLGEKV } \\
\mathrm{l} \mid \mathrm{i}\end{array}$ & FCSRIF \\
\hline $3 M$ & 783 & LIGSP & WC I AMGD & GAREKYAGLGHLCKDYKPC & $\operatorname{TTI}$ \\
\hline Vic-PAV & 315 & & SOCO & $\begin{array}{cc}1: & 1 \\
\text { CDFANEKLEDGMYDHFLOYGE }\end{array}$ & $\begin{array}{l}\text { I } \\
\text { VTE }\end{array}$ \\
\hline
\end{tabular}

Fig. 7. A comparative analysis of luteovirus putative replicase amino acid sequences. An amino acid sequence from the putative BYDV NY-RPV replicase was identified by BESTFIT analysis (Devereux et $a l ., 1984)$ with the SBMV putative replicase amino acid sequence. The NY-RPV region identified was then used in BESTFIT analyses with the amino acid sequence of the putative replicases from BWYV and PLRV. The NY-RPV sequence is represented twice to allow direct comparison with other viruses in the analysis. The comparable region from BYDV Vic-PAV (Miller et al., 1988a) has been included for comparison. Vertical dashes represent identical amino acids and vertical dots indicate chemically related amino acids. Asterisks represent the consensus sequence of RNA-dependent RNA polymerases (Kamer \& Argos, 1984). Boxed regions represent the helicase and replicase motifs identified in NY-RPV ORF3. The Vic-PAV helicase motif VI is located between amino acids 173 and 188 . The numbering represents the amino acid position from the beginning of the ORF in which the GDD sequence is located. RPV, NY-RPV isolate; PLRV (Mayo et al., 1989); BWYV (Veidt et al., 1988); SBMV (Wu et al., 1987); Vic-PAV (Miller et al., 1988a). but similar sequence and gap lengths were found in BWYV (356-SPYFNGKT-38-VFAQED-194) (Veidt et al., 1988).

It has been proposed that the luteovirus viral replicase is expressed as a result of a translational frameshift allowing two overlapping ORFs to produce one protein (Keese et al.,1990; Mayo et al., 1989; Miller et al., 1988a; Veidt et al., 1988). As both ORF2 and ORF3 within the NY-RPV genome are associated with helicase function, it seems probable that both the helicase and the viral replicase are expressed by a translational frameshift mechanism. For the luteovirus Vic-PAV, a region for site-specific ribosomal slippage has been identified within the 16 nucleotide overlap between these ORFs. For other luteoviruses, including NY-RPV, the region of overlap is considerably greater, including up to several hundred nucleotides, but contains no obvious site for ribosome slippage.

\section{Taxonomic considerations}

The genome of the NY-RPV isolate of BYDV shares significant properties with other luteoviruses. These include (i) a replicase-encoding region presumably expressed by translational frameshift, (ii) an ORF, presumably encoding the VPg, completely contained within the $22 \mathrm{~K}$ coat protein gene and (iii) a highly conserved nucleotide sequence surrounding the $22 \mathrm{~K}$ coat protein gene termination codon, which potentially allows the formation of a larger fusion protein. There is also considerable similarity between all luteovirus $22 \mathrm{~K}$ coat proteins, although the NY-RPV coat protein is more like those from BWYV and PLRV than those from the MAV-PS1 and P-PAV isolates of BYDV (Vincent et al., 1990). The greater similarity between the NY-RPV coat protein gene sequence and those of BWYV and PLRV, the similarity between the genome organization of NY-RPV, BWYV and PLRV, and the relative dissimilarity between the genome organization of NY-RPV and Vic-PAV are all consistent with the hypothesis that NY-RPV is a strain of BWYV (Casper, 1988). Hence, NY-RPV should be classified as distinct from BYDV strains similar to Vic-PAV. However, differences in aphid vectors (i.e. R. padi and Myzus persicae) and host range (i.e. monocotyledonous and predominantly dicotyiedonous hosts), suggest that NY-RPV should also be classified as distinct from BWYV and PLRV.

We thank C. Logan for her photographic services and M. Karanjkar for his assistance in the Western blot analysis. This work was funded by Grant 88-37263-3855 from the Competitive Research Grants Office of the U.S. Department of Agriculture and Grant 1484670 from The Quaker Oats Company. The Genetics Computer Group Sequence Analysis Software Package for the VAX was supported by NIH grant no. AI27713. This is Journal Series Article 12706 of the Purdue University Agricultural Experiment Station. 


\section{References}

AAPOLA, A. I. \& Rochow, W. F. (1971). Relationships among three isolates of barley yellow dwarf virus. Virology 46, 127-141.

BaHNER, I., LAMB, J., MAYo, M. A. \& HAY, R. T. (1990). Expression of the genome of potato leafroll virus: readthrough of the coat protein termination codon in vivo. Journal of General Virology 71, 2251-2256.

Barbara, D. J., Kawata, E. E., Ueng, P. P., Lister, R. M. \& LARKINS, B. A. (1987). Production of cDNA clones from the MAV isolate of barley yellow dwarf virus. Journal of General Virology 68 , 2419-2427.

BIRNBOIM, H. C. \& Doly, J. (1979). A rapid alkaline extraction method for screening recombinant plasmid DNA. Nucleic Acids Research 7 , 1513-1523.

CASPER, R. (1988). Luteoviruses. In The Plant Viruses; Polyhedral Virions with Monopartite RNA Genomes, vol. 3, pp. 235-258. Edited by R. Koenig. New York: Plenum Press.

DevereuX, J., Hafberli, P. \& Smithies, O. (1984). A comprehensive set of sequence analysis programs for the VAX. Nucleic Acids Research 12, 387-395.

Gildow, F. E., Ballinger, M. E. \& Rochow, W. F. (1983) Identification of double-stranded RNAs associated with barley yellow dwarf virus infection of oats. Phytopathology 73, 1570-1572.

Gill, C. C. \& Chung, J. (1979). Cytopathological evidence for the division of barley yellow dwarf virus isolates into two subgroups. Virology 95, 59-69.

GorbalenYA, A. E. \& Koonin, E. V. (1989). Viral proteins containing the purine NTP-binding sequence pattern. Nucleic Acids Research 17, 8413-8439.

Gorbalenya, A. E., Koonin, E. V., Donchenko, A. P. \& Blinov, V. M. (1988). A novel superfamily of nucleoside triphosphate binding motif containing proteins which are probably involved in duplex unwinding in DNA and RNA replication and recombination. FEBS Letters 235, 16-24.

HaBILI, N. \& SYMONS, R. (1989). Evolutionary relationship between luteoviruses and other RNA plant viruses based on sequence motifs in their putative RNA polymerases and nucleic acid helicases. Nucleic Acids Research 17, 9543-9555.

HoDGMAN, T. C. (1988a). A new superfamily of replicative proteins. Nature, London 333, 22-23.

HodGman, T. C. (1988 b). Erratum. A new superfamily of replicative proteins. Nature, London 333, 578.

Kamer, G. \& ARgos, P. (1984). Primary structural comparison of RNA-dependent polymerases from plant, animal and bacterial viruses. Nucleic Acids Research 12, 7269-7282.

Kawchuk, L. M., Martin, R. R., Rochon, D. M. \& McPherson, J. (1989). Identification and characterization of the putative potato leafroll virus coat protein gene. Journal of General Virology 70, 783-788.

Keese, P., Martin, R. R., Kawchuk, L. M., Waterhouse, P. M. \& GERLACH, W. L. (1990). Nucleotide sequences of an Australian and a Canadian isolate of potato leafroll luteovirus and their relationships with two European isolates. Journal of General Virology 71, 719-724.

LAEMmLI, U. K. (1970). Cleavage of structural proteins during the assembly of the head of bacteriophage T4. Nature, London 227, 680-685.

Maniatis, T., Fritsch, E. F. \& Sambrook, J. (1982). Molecular Cloning: A Laboratory Manual. New York: Cold Spring Harbor Laboratory

Marsh, L. E., Dreher, T. W. \& Hall, T. C. (1988). Mutational analysis of the core and modulator sequences of the BMV RNA3 subgenomic promoter. Nucleic Acids Research 16, 981-995.

MatThEWs, R. E. F. (1982). Classification and nomenclature of viruses. Intervirology 17, 1-199.

Mayo, M. A., Barker, H., Robinson, D. J., Tamada, T. \& Harrison, B. D. (1982). Evidence that potato leafroll virus RNA is positivestranded, is linked to a small protein and does not contain polyadenylate. Journal of General Virology 59, 163-167.
Mayo, M. A., Robinson, D. J., Jolly, C. A. \& HyMan, L. (1989). Nucleotide sequence of potato leafroll luteovirus RNA. Journal of General Virology 70, 1037-1051.

Miller, W. A., Waterhouse, P. M. \& Gerlach, W. L. (1988a). Sequence and organization of barley yellow dwarf virus genomic RNA. Nucleic Acids Research 16, 6096-6111.

Miller, W. A., Waterhouse, P. M., Kortt, A. A. \& Gerlach, W. L. $(1988 b)$. Sequence and identification of the barley yellow dwarf virus coat protein gene. Virology 165, 306-309.

MuRPHY, J. F., D'ARCY, C. J. \& ClaRK, J. M., JR (1989). Barley yellow dwarf virus RNA has a 5 -terminal genome-linked protein. Journal of General Virology 70, 2253-2256.

Poch, O., Sauvaget, I., Delarue, M. \& Tordo, N. (1989). Identification of four conserved motifs among the RNA-dependent polymerase encoding elements. EMBO Journal 8, 3867-3874.

Prill, B., Maiss, E., Timpe, U. \& Casper, R. (1989). Nucleotide sequence of the potato leafroll virus coat protein gene. Nucleic Acids Research 17, 1768 .

Putney, S. D., Benkovic, S. J. \& Schimmel, P. (1981). A DNA fragment with an $\alpha$-phosphothiolate nucleotide at one end is asymmetrically blocked from digestion by exonuclease III and can be replicated in vivo. Proceedings of the National Academy of Sciences, U.S.A. 78, 7350-7354.

Rochow, W. F. (1970). Barley yellow dwarf virus. CMI/AAB Descriptions of Plant Viruses, no. 32.

Rochow, W. F. (1984). In Barley Yellow Dwarf: Proceedings of the Workshop held at CIMMYT, Mexico, pp. 204-205. Edited by P. A. Burnett. CIMMYT Press.

Rochow, W. F. \& CARMICHAEL, L. E. (1979). Specificity among barley yellow dwarf viruses in enzyme immunosorbent assays. Virology 95 , $415-420$

Rochow, W. F. \& Duffus, J. E. (1981). Luteoviruses and yellows diseases. In Handbook of Plant Virus Infections and Comparative Diagnosis, pp. 147-170. Edited by E. Kurstak. Amsterdam: Elsevier/ North-Holland.

SANGer, F., Nicklen, S. \& Coulson, A. R. (1977). DNA sequencing with chain-terminating inhibitors. Proceedings of the National Academy of Sciences, U.S.A. 74, 5463-5467.

Smith, O. P. \& Harris, K. F. (1990). Potato leaf roll virus $3^{\prime}$ genome organization: sequence of the coat protein gene and identification of a viral subgenomic RNA. Phytopathology 80, 609-614.

TACKe, E., PrüFer, D., Salamini, F. \& Rohde, W. (1990). Characterization of a potato leafroll luteovirus subgenomic RNA: differential expression by internal translation initiation and UAG suppression. Journal of General Virology 71, 2265-2272.

VeIdT, I., Lot, H., Leiser, M., ScheideCKer, D., Guilley, H., RICHARDS, K. \& JoNARD, G. (1988). Nucleotide sequence of beet western yellows virus RNA. Nucleic Acids Research 16, 9917 9932.

Vieira, J. \& Messing, J. (1982). The pUC plasmids, an m13mp7derived system for insertion mutagenesis and sequencing with universal primers. Gene 19, 259-268.

Vincent, J. R., Ueng, P. P., Lister, R. M. \& LaRkins, B. A. (1990). Nucleotide sequences of coat protein genes for three isolates of barley yellow dwarf virus and their relationships to other luteovirus coat protein sequences. Journal of General Virology 71, 27912799.

Waterhouse, P. M., Gildow, F. E. \& Johnstone, G. R. (1988). Luteovirus group. AAB Descriptions of Plant Viruses, no. 339.

Wu, S., Rinehart, C. A. \& Kaesberg, P. (1987). Sequence and organization of southern bean mosaic virus genomic RNA. Virology 161, 73-80.

YounG, R. A. \& DAvIs, R. W. (1983). Efficient isolation of genes using antibody probes. Proceedings of the National Academy of Sciences, U.S.A. 80, 1194-1198.

(Received 27 February 1991; Accepted 10 June 1991) 